\title{
Unambiguous Photometric Stereo Using Two Images
}

\author{
Roberto Mecca ${ }^{1}$ and Jean-Denis Durou ${ }^{2}$ \\ 1 Dipartimento di Matematica "G. Castelnuovo" \\ Sapienza - Universitá di Roma \\ roberto.mecca@mat.uniroma1.it \\ Www.mat.uniroma1.it/ mecca \\ 2 IRIT - Université Paul Sabatier - Toulouse \\ Jean-Denis.Durou@irit.fr
}

\begin{abstract}
In the last years, the 3D reconstruction of surfaces which represent objects photographed by simple digital cameras has become more and more necessary to the scientific community. Through the most various mathematical and engineering methods, scientists continue to study the Shape-from-shading problem, using the photometric stereo technique which allows the use of several light sources, but keeps the camera at the same point of view. Several studies, through different advances on the problem, have checked that in the applications, the smallest number of photos that have to be considered is three. In this article we analyze the possibility to determine the objects' surface using two images only.
\end{abstract}

Keywords: Shape-from-shading, photometric stereo, normals integration, PDE numerical analysis, boundary conditions.

\section{Introduction}

Many articles have been written about the impossibility to solve the Shapefrom-shading problem (SFS) considering only one picture [1, even if a recent perspective SFS model exploiting the attenuation of the lighting with respect to the distance to the light source has been shown to yield to a well-posed problem, if complemented by reasonable assumptions 2. This impossibility, from the PDEs point of view, comes out from the difficulty we meet in differentiating the concave surfaces from the convex ones. The most natural way to solve the problem is to use more than one picture. First introduced by Woodham [3], photometric stereo (PS) consists in using several images which portray the object photographed always from the same point of view, but with different light source positions 4].

There are two main approaches to solve the SFS-PS problem. The first one aims at computing in each point the normal to the surface to be reconstructed. If the albedo is supposed to be known, this approach has the drawback to be well-posed only if a minimum of three images of a differentiable surface are used (we emphasize that the required regularity of the surface, in real applications, 
can be seen as a supplementary disadvantage). Its vantage is that, even if only two images are used, the number of solutions of the problem (for a differentiable surface) is a priori predictable through the study that we propose in this work.

The second approach, which is more recent, aims at solving the PDE model. If we still suppose the albedo to be known, it has the advantage of admitting only one solution even if only two images are used. It is also possible to approximate the solution even if the surface is Lipschitzian (that is, almost everywhere differentiable). The drawback is that it is well-posed only if we preliminary know the height of the surface on the boundary of the image (i.e. only if we know the boundary condition of the differential problem).

The main idea of this paper is to approximate the boundary condition by integrating the normal field only on the boundary of the image, and then to solve the PS problem anywhere else using the PDE approach. In Section 2, we recall the differential and non-differential formulations of SFS-PS. In Section 3 , we show that in some points, two images are enough to deduce the normal univocally. Section 4 is dedicated to the tests and Section 5 to conclusion and perspectives.

\section{Main Features of the Photometric Stereo Technique}

\subsection{Shape-from-Shading}

We start by giving a brief outline of the SFS problem and introducing the basic assumptions. We attach to the camera a 3D Cartesian coordinate system $x y z$, such that $x y$ coincides with the image plane and $z$ with the optical axis. Under the assumption of orthographic projection, the visible part of the surface is a graph $z=u(x, y)$. For a Lambertian surface of uniform albedo equal to 1 , lighted by a unique light source located at infinity in a direction indicated by the unitary vector $\omega=\left(\omega_{1}, \omega_{2}, \omega_{3}\right)=\left(\tilde{\omega}, \omega_{3}\right) \in \mathbb{R}^{3}$, the SFS problem can be modeled by the following "image irradiance equation" [5]:

$$
n(x, y) \cdot \omega=I(x, y) \quad \forall(x, y) \in \bar{\Omega}
$$

where $I(x, y)$ is the greylevel at the image point $(x, y)$ and $n(x, y)$ is the unitary outgoing normal to the surface at the scene point $(x, y, u(x, y))$. The greylevel $I$, which is the datum in the model, is assumed to take real values in the interval $[0,1]$. The height $u$, which is the unknown, has to be reconstructed on a compact domain $\bar{\Omega}=\Omega \cup \partial \Omega \subset \mathbb{R}^{2}$ called the "reconstruction domain". It does not explicitely appear in Eq. (1), but implicitely through the normal $n(x, y)$, since this vector can be written:

$$
n(x, y)=\frac{1}{\sqrt{1+|\nabla u(x, y)|^{2}}}[-\nabla u(x, y), 1]^{\top}
$$

Combining Eqs. (11) and (2), we arrive to the following differential formulation of the SFS problem:

$$
\frac{-\nabla u(x, y) \cdot \widetilde{\omega}+\omega_{3}}{\sqrt{1+|\nabla u(x, y)|^{2}}}=I(x, y) \quad \forall(x, y) \in \Omega
$$


which is a first order non-linear PDE of the Hamilton-Jacobi type. Eq. (3) with the add of a Dirichlet boundary condition $u(x, y)=g(x, y) \forall(x, y) \in \partial \Omega$, do not admit a unique solution if the brightness function $I$ reaches its maximum i.e., if there are points $(x, y) \in \Omega$ such that $I(x, y)=1$. In this case, we cannot distinguish whether a surface is concave or convex ("concave/convexity ambiguity", see [5]).

With the purpose to prove the existence of a unique solution, we increase the information about the surface considering the photometric stereo technique.

\subsection{Photometric Stereo: Differential Approach}

The first approach to PS is based on the differential formulation (3) of the SFS problem, that is, using two images we have:

$$
\left\{\begin{aligned}
\frac{-\nabla u(x, y) \cdot \widetilde{\omega}+\omega_{3}}{\sqrt{1+|\nabla u(x, y)|^{2}}}=I(x, y) & \text { a.e. }(x, y) \in \Omega \\
\frac{-\nabla u(x, y) \cdot \widetilde{\omega}^{\prime}+\omega_{3}^{\prime}}{\sqrt{1+|\nabla u(x, y)|^{2}}}=I^{\prime}(x, y) & \text { a.e. }(x, y) \in \Omega \\
u(x, y)=g(x, y) & \forall(x, y) \in \partial \Omega
\end{aligned}\right.
$$

It is a PDEs non-linear system with the add of a Dirichlet boundary condition that admits a unique solution in the space of Lipschitzian functions. This means that, even if a surface is differentiable for almost every $(x, y) \in \bar{\Omega}$ it is possible to approximate it using a convergent numerical scheme [6]. The only drawback of this formulation concerns the boundary condition knowledge. In fact, beyond the image data $\left(I, I^{\prime}\right)$ and the light vectors $\left(\omega, \omega^{\prime}\right), g(x, y)$ (taken in the space of the Lipschitz functions $W^{1, \infty}(\partial \Omega)$ ) represents an additional information that we must know to make this approach work.

Let us explain how the differential approach works. To arrive to the final PDE formulation, we simplify the system (4) eliminating its non-linearity, supposing that $1 \geq I(x, y)>0$ everywhere. That is, we consider for example the following equality from the first equation:

$$
\sqrt{1+|\nabla u(x, y)|^{2}}=\frac{-\nabla u(x, y) \cdot \widetilde{\omega}+\omega_{3}}{I(x, y)}
$$

and replacing (5) into the other equation we obtain a linear equation, $\forall(x, y) \in \Omega$ :

$$
\left[I^{\prime}(x, y) \omega_{1}-I(x, y) \omega_{1}^{\prime}\right] \frac{\partial u}{\partial x}+\left[I^{\prime}(x, y) \omega_{2}-I(x, y) \omega_{2}^{\prime}\right] \frac{\partial u}{\partial y}=I^{\prime}(x, y) \omega_{3}-I(x, y) \omega_{3}^{\prime}
$$

Considering also the same boundary condition as that of (4), it is possible to arrive to the following linear problem:

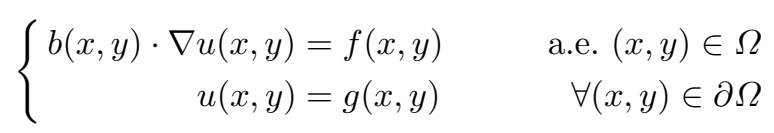


where

$$
\left\{\begin{array}{l}
b(x, y)=\left[I^{\prime}(x, y) \omega_{1}-I(x, y) \omega_{1}^{\prime}, I^{\prime}(x, y) \omega_{2}-I(x, y) \omega_{2}^{\prime}\right]^{\top} \\
f(x, y)=I^{\prime}(x, y) \omega_{3}-I(x, y) \omega_{3}^{\prime}
\end{array}\right.
$$

With these elements it is possible to enunciate the following result [6]:

Theorem 1. Let $b(x, y)$ and $f(x, y)$ be both bounded functions defined by (8)), where $I$ and $I^{\prime}$ are two greylevel functions such that $1 \geq I, I^{\prime}>0$, with a jump discontinuity on the piecewise regular curve $\gamma(s)$ and $g(x, y) \in W^{1, \infty}(\partial \Omega)$. If $\gamma(s)$ is not a characteristic curve of the problem (7) then it admits a unique Lipschitzian solution $u(x, y)$.

\subsection{Photometric Stereo: Non-differential Approach}

The other approach is based on the local estimation of the outgoing unitary normal to the surface. With the same data as before, Eq. (1) gives the following non-linear system in the coordinates of the normal, in each point $(x, y) \in \bar{\Omega}$ :

$$
\left\{\begin{aligned}
n_{1}(x, y)^{2}+n_{2}(x, y)^{2}+n_{3}(x, y)^{2} & =1 \\
\omega_{1} n_{1}(x, y)+\omega_{2} n_{2}(x, y)+\omega_{3} n_{3}(x, y) & =I(x, y) \\
\omega_{1}^{\prime} n_{1}(x, y)+\omega_{2}^{\prime} n_{2}(x, y)+\omega_{3}^{\prime} n_{3}(x, y) & =I^{\prime}(x, y)
\end{aligned}\right.
$$

This approach goes on with the integration of the normal field using Eq. (2), all over the domain $\bar{\Omega}$ [7]. Its drawback is that the non-linear system (9) has not a unique solution in general.

The purpose of our work is to find and, in particular, characterize the zones of the images where the solution to (9) is unique. This permits us to understand, before the integration of the gradient field, the number of possible surfaces approximated by this approach. With the aim to combine both approaches, we study in detail the problem (9) giving information about all possible solutions. We advance that there are two local solutions at the most, but the problem is that they can globally generate much more than two surfaces.

\section{Photometric Stereo with 2 Images: Normal Uniqueness}

\subsection{General Study of the Problem}

We now focus on the problem of normal estimation, emphasizing one more time that it is based on a local study of the images. For each pixel we want to estimate the unitary vector which represents the outgoing normal to the surface. We can determine the set of visible normals as the superior part of a sphere centered at the origin and with radius one, that is the Northern hemisphere of the so-called Gaussian sphere $\mathcal{S}$ (see Fig. 10). Referring to the 3D Cartesian coordinate system $x y z$, these vectors $n=\left[n_{1}, n_{2}, n_{3}\right]^{\top}$ are those such that $n_{3}>0$. We now consider the two light vectors $\omega$ and $\omega^{\prime}$ and the sets of lighted normals, that is those such 
that $\omega \cdot n>0$ and $\omega^{\prime} \cdot n>0$. On $\mathcal{S}$, these sets are limited by the two planes $\pi$ and $\pi^{\prime}$ passing through the origin and orthogonal to $\omega$ and $\omega^{\prime}$. Now, we can determine the set of possible normals for each twice-lighted point $(x, y)$, which can be summed up in the following non-linear system:

$$
\left\{\begin{aligned}
n_{1}(x, y)^{2}+n_{2}(x, y)^{2}+n_{3}(x, y)^{2} & =1 \\
\omega_{1} n_{1}(x, y)+\omega_{2} n_{2}(x, y)+\omega_{3} n_{3}(x, y) & \geq 0 \\
\omega_{1}^{\prime} n_{1}(x, y)+\omega_{2}^{\prime} n_{2}(x, y)+\omega_{3}^{\prime} n_{3}(x, y) & \geq 0 \\
n_{3}(x, y) & \geq 0
\end{aligned}\right.
$$

On the other hand, in each twice-lighted point $(x, y)$, the linear system (9) usually admits two solutions. An important study is carried out on the straight line $\Delta=\pi^{\prime} \cap \pi^{\prime \prime}$, which is supported by the vector $r=\omega \times \omega^{\prime}$. This allows us to establish a direct connection between the solutions of (9). For each twice-lighted point $(x, y)$ such that (9) admits two solutions $\hat{n}$ and $\hat{\hat{n}}$, the locations of these normals on $\mathcal{S}$ define a straight line which is parallel to $\Delta$ since, according to the Lambertian model, $\hat{n}$ and $\hat{\hat{n}}$ form the same angles with $\omega$ and $\omega^{\prime}$ (see Fig. 1).

\subsection{Normal Uniqueness Obtained by Visibility or by Coincidence}

The first case in which normal uniqueness using two images we can be proved is explainable geometrically, considering the set obtained changing the sign of the last inequality of the system (10) (which represents the condition of visibility) and then projecting it, according to the direction $r$, on the other side of the sphere. Therefore, in order to determine these points in the images, the first step is to determine the set $\Omega_{G} \subset \bar{\Omega}$ of points $(x, y)$ such that there exists a solution $\hat{n}$ to the following system:

$$
\left\{\begin{aligned}
n_{1}(x, y)^{2}+n_{2}(x, y)^{2}+n_{3}(x, y)^{2} & =1 \\
\omega_{1} n_{1}(x, y)+\omega_{2} n_{2}(x, y)+\omega_{3} n_{3}(x, y) & \geq 0 \\
\omega_{1}^{\prime} n_{1}(x, y)+\omega_{2}^{\prime} n_{2}(x, y)+\omega_{3}^{\prime} n_{3}(x, y) & \geq 0 \\
n_{3}(x, y) & <0
\end{aligned}\right.
$$

Clearly, these normals $\hat{n}$ cannot be considered as possible candidates for the normal field of the surface taken into consideration, because they are located on the non-visible part of $\mathcal{S}$ (see the yellow part $\mathcal{S}_{Y}$ of $\mathcal{S}$ in Fig. 1). The normals which could be candidate for the normal field are those in the set in biunique correspondence with $\mathcal{S}_{Y}$ with respect to the direction $r$ (see the green part $\mathcal{S}_{G}$ of $\mathcal{S}$ in Fig. 1). Note that, if $\omega$ or $\omega^{\prime}$ is vertical i.e., equal to $[0,0,1]^{\top}$, then $r$ is horizontal and, therefore, the set $\Omega_{G}$ is empty.

The second way of obtaining normal uniqueness corresponds to the limit case where the two solutions $\hat{n}$ and $\hat{\hat{n}}$ of (9) coincide. The set $\Omega_{R} \subset \bar{\Omega}$ thus contains the points $(x, y)$ where the normal is orthogonal to the direction $r$ (see the red line $\mathcal{S}_{R}$ on $\mathcal{S}$ in Fig. 2), which is a geodesic line on $\mathcal{S}$ between two extreme points $\hat{P}$ and $\hat{P}^{\prime}$.

Looking at Figs. 1] and 2, it is obvious that $\mathcal{S}_{R} \cap \mathcal{S}_{G}=\emptyset$ as soon as $\omega_{3}>0$ and $\omega_{3}^{\prime}>0$, which implies that $\Omega_{G} \cap \Omega_{R}=\emptyset$ as well. 


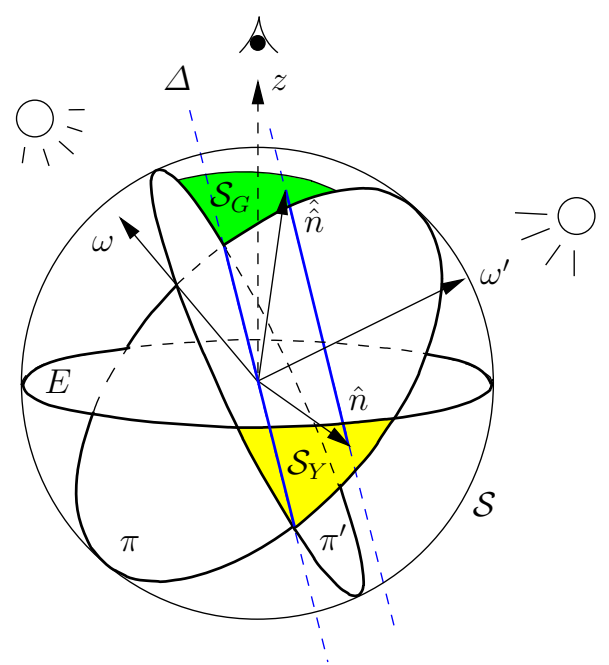

Fig. 1. The planes $\pi$ and $\pi^{\prime}$ are orthogonal, respectively, to the light vectors $\omega$ and $\omega^{\prime}$. The intersection between $\pi$ and $\pi^{\prime}$ is denoted as $\Delta$. Each normal $\hat{\hat{n}}$ pointing to the green area $\mathcal{S}_{G}$ is known without ambiguity, since the second possible normal $\hat{n}$ points to the yellow area $\mathcal{S}_{Y}$, which is a twice lighted but non-visible part of $\mathcal{S}$ (because it lies under the equator $E$ ).

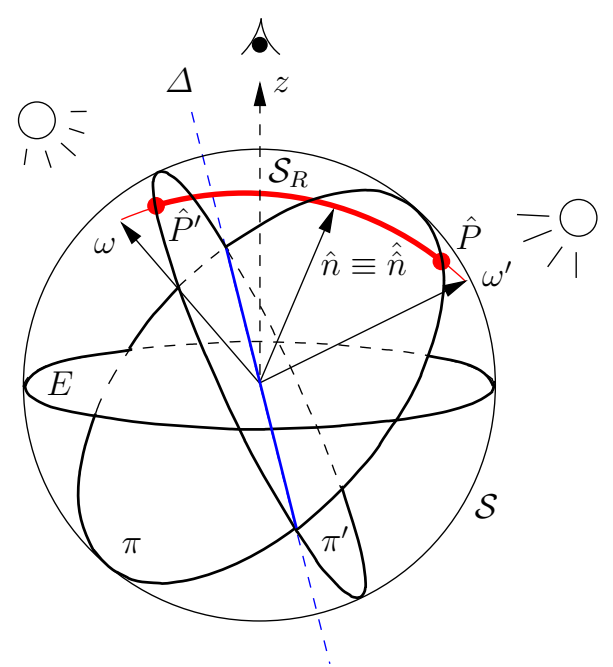

Fig. 2. The geodesic $\mathcal{S}_{R}$ in red is a part of the intersection between $\mathcal{S}$ and the plane supported by $\omega$ and $\omega^{\prime}$, limited by $\hat{P}$ and $\hat{P}^{\prime}$. Each normal pointing to $\mathcal{S}_{R}$ is known without ambiguity, since both normals $\hat{n}$ and $\hat{\hat{n}}$ coincide in this case. 


\subsection{Finding the Sets $\Omega_{G}$ and $\Omega_{R}$}

Here we describe how to find the sets $\Omega_{G}$ and $\Omega_{R}$ in the reconstruction domain $\bar{\Omega}$. For each normal $\tilde{n}=\left[\tilde{n}_{1}, \tilde{n}_{2}, \tilde{n}_{3}\right]^{\top} \in \mathcal{S}_{G} \cup \mathcal{S}_{R}$, we have to calculate the correspondent couple of greylevels $\left(\tilde{I}, \tilde{I}^{\prime}\right)$ using (1) , i.e.:

$$
\left\{\begin{array}{c}
\tilde{I}=\omega_{1} \tilde{n}_{1}+\omega_{2} \tilde{n}_{2}+\omega_{3} \tilde{n}_{3} \\
\tilde{I}^{\prime}=\omega_{1}^{\prime} \tilde{n}_{1}+\omega_{2}^{\prime} \tilde{n}_{2}+\omega_{3}^{\prime} \tilde{n}_{3}
\end{array}\right.
$$

and check if, for each pixel $(i, j) \in \bar{\Omega}$, the greylevels $\left(I_{i, j}, I_{i, j}^{\prime}\right)$ are such that:

$$
\left\{\begin{array}{l}
\left|I_{i, j}-\tilde{I}\right|<\varepsilon \\
\left|I_{i, j}^{\prime}-\tilde{I}^{\prime}\right|<\varepsilon
\end{array}\right.
$$

for a small fixed value of $\varepsilon$ ( $\varepsilon=0.001$ is used in the tests).

Definition 2. Given a pair of images, we call $\Omega_{G}^{p}$ and $\Omega_{R}^{p}$ the sets of pixels of $\bar{\Omega}$ which belong to $\Omega_{G}$ and $\Omega_{R}$ and are determined using the criterion (13).

As we will see in the numerical tests, the sets $\Omega_{G}^{p}$ and $\Omega_{R}^{p}$, depending on the shape of the surface, can be made of several disjoint parts, that is $\Omega_{G}^{p}=\Omega_{G}^{p}(1) \cup$ $\ldots \cup \Omega_{G}^{p}\left(n_{G}\right)$ and $\Omega_{R}^{p}=\Omega_{R}^{p}(1) \cup \ldots \cup \Omega_{R}^{p}\left(n_{R}\right)$.

\subsection{Predictability of the Number of Global Solutions}

Let us suppose that the system (9) always admits two solutions $\hat{n}$ and $\hat{\hat{n}}$. In fact, (9) could have no solution in some points where the greylevels do not perfectly match the Lambertian model. Nevertheless, we know under this assumption that there exist either one or two possible normals in each twice-lighted point $(x, y) \in$ $\bar{\Omega}$. If moreover the surface to be reconstructed is supposed to be differentiable everywhere, then the number of global normal fields is predictable. For example, if $\Omega_{R}^{p}$ is empty while $\Omega_{G}^{p}$ is not empty, then the normal field is unique, since all the normals point toward the twice-lighted part $\mathcal{S}_{U}$ of $\mathcal{S}$ which lies between $\mathcal{S}_{G}$ and $\mathcal{S}_{R}$ (see Figs. 1 and 2). More generally, this analysis of the problem allows us to predict the number of global solutions.

Another interesting advantage one can take from the study of the sets $\Omega_{G}^{p}$ and $\Omega_{R}^{p}$ is related to the PDE approach (7). For example, let us suppose that no pixel lying on the boundary $\partial \Omega$ belongs to $\Omega_{R}^{p}$. This means that there are two different normal fields along the boundary i.e., two different values for $\nabla u$ according to (21), and finally two boundary conditions $u(x, y)=g(x, y)$ considering:

$$
g(\beta(t))=g_{1}(\beta(0))+\int_{0}^{t} \nabla u(\beta(s)) \beta^{\prime}(s) d s
$$

where $\beta(t)$ is a parametrization of $\partial \Omega$. Then, according to Theorem 1, we can approximate the height of the surface with two values in this case (supposing one more time that the surface is differentiable on the boundary $\partial \Omega$ ). 


\section{Numerical Tests}

Here we present some numerical tests on the synthetic surfaces shown in Fig. 3 , the surface on the left is differentiable everywhere, whereas that on the right is only Lipschitzian (differentiable almost everywhere).
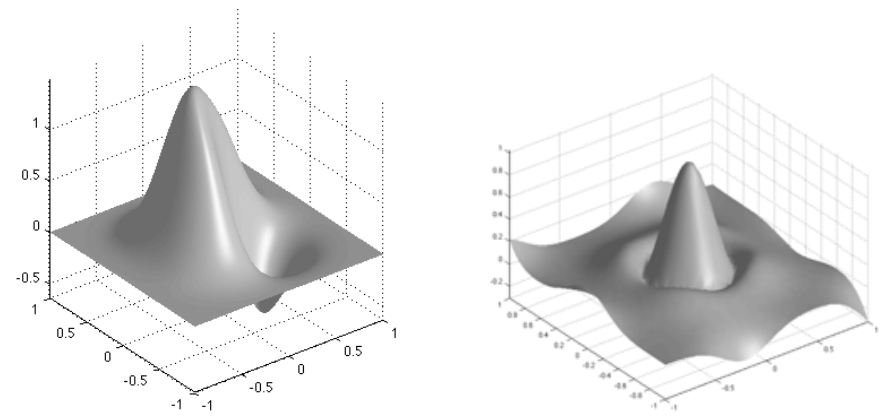

Fig. 3. Surfaces $S_{\text {Regular }}$ (left) and $S_{\text {Lipschitz }}$ (right) used in the numerical tests

\subsection{Let's Count the Solutions!}

A first example uses the pair of images of $S_{\text {Regular }}$ shown in Fig. 4, over which the two sets $\Omega_{G}^{p}$ and $\Omega_{R}^{p}$ are superimposed. Below each image, the spherical coordinates $(\varphi, \theta)$ of the light vector, such that $\omega=(\sin \varphi \cos \theta, \sin \varphi \sin \theta, \cos \varphi)$, are given.

In order to understand how to count the solutions, let us introduce the sets $\mathcal{S}_{U}$ and $\mathcal{S}_{D}$, which are the subsets of the Gaussian sphere lying, respectively, upon and below the geodesic $\mathcal{S}_{R}$.
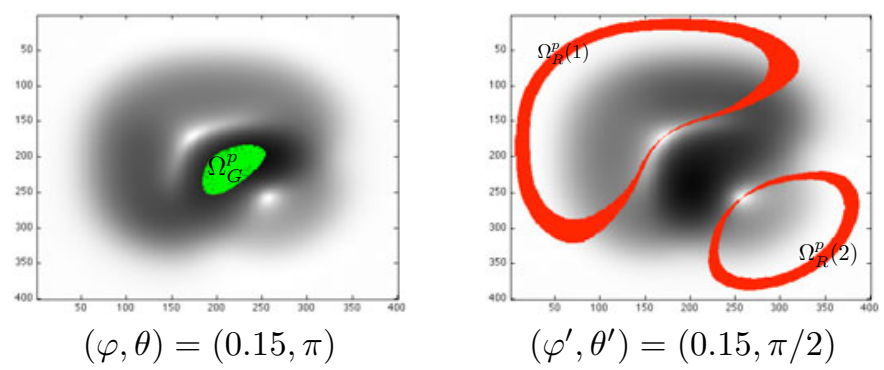

Fig. 4. Pair of synthetic images of the surface $S_{\text {Regular }}(400 \times 400$ pixels $)$ used to count the solutions. Note that all the pixels are twice-lighted.

Now, taking into account that these sets can be mapped to the reconstruction domain $\bar{\Omega}$ (namely $\Omega_{U}^{p}=\Omega_{U}^{p}(1) \cup \ldots \cup \Omega_{U}^{p}\left(n_{U}\right)$ and $\Omega_{D}^{p}=\Sigma_{D}^{p}(1) \cup \ldots \cup \Omega_{D}^{p}\left(n_{D}\right)$ ), we show in Fig. 5 the four solutions deduced from the sets previously described. 

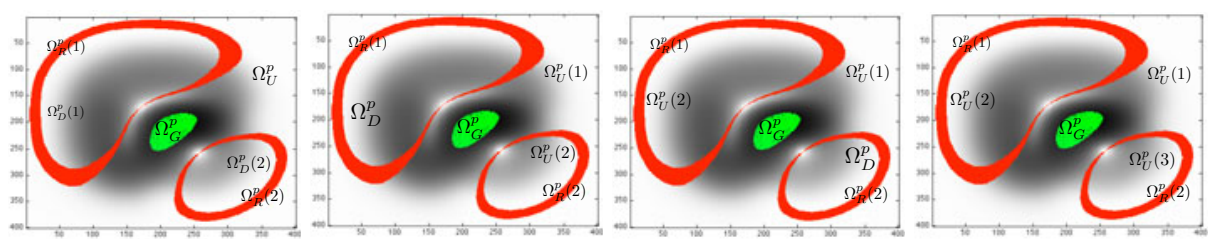

Fig. 5. All possible combinations that allow us to predict the number of solutions

\subsection{Combining the Differential and Non-differential Approaches}

A second example uses the pair of images of $S_{\text {Lipschitz }}$ shown in Fig. 6, over which the set of points where the surface is not differentiable is superimposed in blue. A problem that we want to avoid is the presence of pixels of $\Omega_{R}^{p}$ on the boundary $\partial \Omega$, since this would give rise to an ambiguity. In fact, in each pixel of $\Omega_{R}^{p}$, the normal can cross the geodesic $\mathcal{S}_{R}$, passing from $\mathcal{S}_{D}$ to $\mathcal{S}_{U}$ (or vice versa) or remain on the same side of $\mathcal{S}_{R}$. With this aim we choose the light vectors $\omega$ and $\omega^{\prime}$ very close to each other, in order to reduce the sizes of $\mathcal{S}_{G}$ and $\mathcal{S}_{R}$, and therefore to reduce those of $\Omega_{G}^{p}$ and $\Omega_{R}^{p}$. As in the first test, if some pixels of $\partial \Omega$ belong to $\Omega_{R}^{p}$, then we are able to count the different boundary conditions. In our example, we find that $\Omega_{G}^{p}=\emptyset$ and $\Omega_{R}^{p}=\emptyset$. This means that there are only two possible boundary conditions, but only one is admissible for a correct approximation of the surface. The remaining question is thus: is it possible to find the correct boundary condition?

Once the two possible normal fields are computed along $\partial \Omega$, we can integrate them using a very simple method which consists in fixing the height in some reference pixel and then computing the height along $\partial \Omega$ as Strat did (see [8]). It is well-known that the integration of an irrotational field along a closed contour is equal to zero [7. Therefore, it is possible to decide which boundary condition is the right one, just comparing both integrals. In our example, we find an $L^{\infty}(\partial \Omega)$
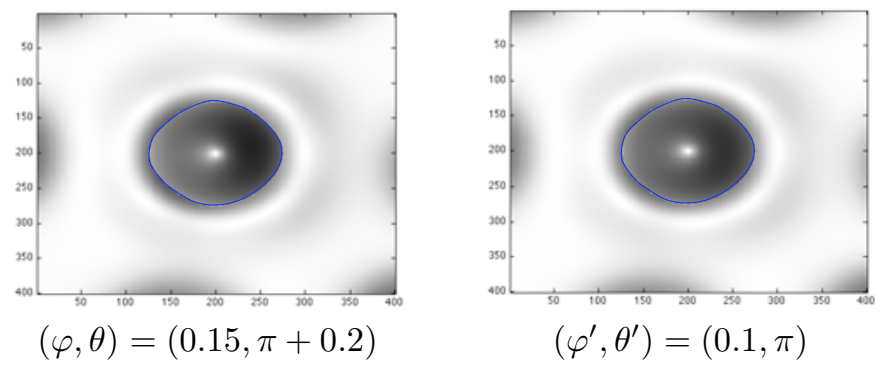

Fig. 6. Pair of synthetic images of the surface $S_{\text {Lipschitz }}(400 \times 400$ pixels $)$ used for the approximation of the boundary condition. In blue is emphasized the set of points where the surface is not differentiable. 
norm error on $\partial \Omega$ between the real and the predicted boundary conditions equal to $5.38 \times 10^{-3}$ for $S_{\text {Regular }}$ and to $3.325 \times 10^{-2}$ for $S_{\text {Lipschitz }}$. Unfortunately, due to the lack of space, we cannot show here the whole 3D-reconstructions obtained using the PDE approach (7).

\section{Conclusion and Perspectives}

In this paper, we addressed photometric stereo using two images only. This particular situation is rarely studied because using more than three images usually suffices to render the problem well-posed. Nevertheless, there are at least two reasons which can validate our work. First, the situation where all the light vectors are coplanar is known to be ill-posed, and reduces to the case with two lights only. Note that this is exactly the case of an outdoors scene lighted by the Sun. Second, the "standard" PS technique supposes that the albedo of the scene is unknown, in order to linearize the problem, but this is not always necessary and can moreover give rise to inaccuracies in the reconstructions. From that point of view, our work is an interesting insight in the non-linear PS problem.

Our main result is to show that the number of solutions of PS using two images is predictable, thanks to particular points where the normal can be estimated without ambiguity. More tests have to be performed in order to more clearly show the accuracy of our approach, since this work was rather theoretical. In addition, it must be questionned to which extent our results could be useful to standard PS, since $n$ images induce $C_{2}^{n}$ pairs of images!

\section{References}

1. Durou, J.-D., Falcone, M., Sagona, M.: Numerical Methods for Shape-from-shading: A New Survey with Benchmarks. CVIU 109(1), 22-43 (2008)

2. Prados, E., Faugeras, O., Camilli, F.: Shape from Shading: a well-posed problem? Rapport de Recherche 5297, INRIA, Sophia Antipolis, France (August 2004)

3. Woodham, R.J.: Photometric Method for Determining Surface Orientation from Multiple Images. Optical Engineering 19(1), 139-144 (1980)

4. Kozera, R.: Existence and Uniqueness in Photometric Stereo. Appl. Math. Comp. 44(1), 1-103 (1991)

5. Horn, B.K.P., Brooks, M.J.: Shape from Shading. MIT Press, Cambridge (1989)

6. Mecca, R.: A constructive differential approach to photometric shape-from-shading (2010) (preprint)

7. Durou, J.-D., Aujol, J.-F., Courteille, F.: Integrating the Normal Field of a Surface in the Presence of Discontinuities. In: Cremers, D., Boykov, Y., Blake, A., Schmidt, F.R. (eds.) EMMCVPR 2009. LNCS, vol. 5681, pp. 261-273. Springer, Heidelberg (2009)

8. Horn, B.K.P., Brooks, M.J.: The Variational Approach to Shape From Shading. CVGIP 33(2), 174-208 (1986) 